\title{
Glycosidases during chick embryo lung development and their colocalization with proteoglycans and growth factors
}

\author{
G. Stabellini, M. Calvitti ${ }^{1}$, T. Baroni ${ }^{1}$, L. Marinucci ${ }^{1}$, C. Calastrini ${ }^{2}$, P. Carinci ${ }^{3}$ and E. Becchetti ${ }^{1}$ \\ Dipartimento di Anatomia Umana, Università Statale di Milano; 'Dipartimento di Medicina Sperimentale e Scien- \\ ze Biochimiche, Università di Perugia; ${ }^{2}$ Dipartimento di Morfologia ed Embriologia, Università di Ferrara and ${ }^{3}$ Isti- \\ tuto di Istologia ed Embriologia, Università di Bologna, Italy
}

Accepted: 12/11/2001

Key words: epithelial branching development, extracellular matrix, ß-hexosaminidase, TGFß2, IL-1.

\section{SUMMARY}

During development, the epithelial component of the lung goes through a complex orderly process of branching, following strict patterns of space and time. Proteoglycans, glycosaminoglycans and growth factors are fundamental components of the extracellular matrix and perform a key role in differentiative processes. The embryonic chick lung shows a specific glycosaminoglycan composition at different levels of branching and at different embryonic stages. Proteoglycan and glycosaminoglycan accumulation is the result of secretion, absorption and degradation processes. In this pathway, enzymes, such as glycosidases, growth factors and cytokines are involved. We examined the behaviour of glycosidases, such as $\beta$-hexosaminidases ( $\beta$ - $\mathrm{N}$-acetyl-D-glucosaminidase, $\beta$ $\mathrm{N}$-acetyl-D-galactosaminidase), B-glucuronidase and B-galactosidase, during the development of the lung bud. Our data show that the activity of the enzymes is closely linked to the processes of epithelial proliferation, bronchial tubule lengthening and infiltration of the surrounding mesenchyme. The glycosaminoglycans colocalize with transforming growth factor $\beta_{2}$ and inter- leukin-1 in the basement membrane and in the mesenchymal areas where the epithelium grows, and are complementary to the presence of the glycosidases. In conclusion, the activity of these glycosidases is spatially and temporally programmed and favors the release of the factors and the events which they influence.

\section{INTRODUCTION}

The regulation of morphogenesis in various organs such as lung, kidney, skin and limbs (Becchetti et al., 1988; Davies et al., 1995, Kulyk and Kosher, 1987; Stabellini et al., 1998) is the result of complex processes in which the regulation of the extracellular matrix (ECM) plays a key role. The control of ECM composition has therefore to be considered as a main aspect for both cell strategy and the influence of environmental factors on cellular behavior.

For instance, growth factors such as transforming growth factor $\alpha$ (TGF $\alpha$ ), transforming growth factor $\beta$ (TGFß), interleukin-1 (IL-1) have been shown to control the accumulation of ECM components such as glycosaminoglycans (GAG) and 
proteoglycans (PG) (Locci et al., 1993; Bodo et al., 1994; Evangelisti et al., 1998). This process requires a continuous remodelling involving deposition, degradation and absorption processes. Glycosidases (exo- and endoglycosidases) are the lysosomal enzymes mainly involved in the degradation of GAG (Kresse and Glössl, 1987).

Exoglycosidases hydrolyze glycoside linkages at the non-reducing terminus of the chain involving different glucoside residues. The activity exerted by exoglycosidases differs according to the individual GAG: $\beta$-N-acetyl-D-glucosaminidase (B-NAG) acts on hyaluronic acid (HA) and keratan sulphate (KS); B-N-acetyl-D-galactosaminidase (B-NAcGALA) acts on chondroitin 4 and 6 sulphate (CS) and dermatan sulphate (DS); ß-glucuronidase ( $\beta$-GLU) acts on CS, DS, and heparan sulphate (HS); B-D-galactosidase (ß-GALA) acts on KS and carbohydrateprotein linkage of sulphated GAG. Endoglycosidases such as hyaluronidase (HAase) act on HA and CS. In embryonic chick fibroblasts, HAase was found in both the cells and the culture medium (Kresse and Glössl, 1987).

In vivo, qualitative and quantitative GAG composition of the ECM changes during lung development, showing a different distribution as regards bronchial branching (Becchetti et al., 1988). In particular, morphological and histochemical reconstructions demonstrate that, on days 6-8, HA prevails around the mesobronchus and CS dominates $2^{\text {nd }}-$ order branching, whereas on days 9-12 CS increases in the ground substance of mesenchyme. This increase is irregular, being smaller in older areas (mesobronchus and $2^{\text {nd }}$ order bronchi) and larger in the most recent $3^{\text {rd }}$ order bronchi (parabronchi), which extend into the lateral and dorsal areas of the lung rudiment. On days 13-18, GAG are uniformly distributed in the mesenchymal septa and around the interlobular vascular network. It has also been demonstrated that they increase throughout the lung until developmental day 14 and then decrease until day 18 (Carinci, 1981).

It has been shown that the ECM has heterogeneous composition and, by the addition of exogenous GAG or by enzymatic digestion, its correlation with changes to the bronchial tree has been demonstrated (Carinci et al., 1986). Therefore, the morphogenesis of epithelial branching proves to be a suitable model for studying the regulatory role of ECM and of the factors conditioning its rearrangement.
In this study, we first intended to explain the spatial and temporal correlation between epithelial branching and glycosidase activity. To this end, we performed enzymatic assays either in the lung in toto or in three different regions (apical, medial and caudal) along the distal axis where the bronchial branching shows significant differences, as previously demonstrated (Becchetti et al., 1988).

We then determined, by immunohistochemical analysis, the colocalization of B-NAG, HA or PG and $\mathrm{TGFB}_{2}$ or IL-1 in a section of the medial region at a critical time for bronchial branching development, when $3^{\text {rd }}$ order bronchi (ecto and entobronchi) emerge (9 days of developmental stage). Since fibroblasts are involved in the deposition and adsorption of ECM, we verified the specific behaviour of glycosidase variations in fibroblasts derived from chick embryo lungs at different stages of development and cultured in medium without serum.

Our data show a clear correlation between epithelial branching and the dynamics of GAG accumulation, allowing us to suggest a regulatory model in interactions between the glycosidases, GAG, PG and the growth factors and cytokines involved. Moreover, glycosidase activity in embryonic chick lung fibroblasts at different stages of development confirms that the enzymes are chronologically related to lung development.

\section{MATERIALS AND METHODS}

Lung rudiments were carefully removed from chick embryos at incubation days 7, 911,13 , and 18 according to the Hamburger- Hamilton table (Hamilton, 1965).

Lung rudiments were dissected with fine knives into the apical, medial and caudal regions according to previous studies on the spatial distribution of GAG in chick embryo lung (Becchetti et al., 1988). Whole-lung, apical, medial and caudal portions were pooled, washed with $0.15 \mathrm{M} \mathrm{NaCl}$ at $4^{\circ} \mathrm{C}$ and employed for enzyme determination.

For histochemical localization of glycosidases, colocalization of glycosidases-GAG and cytokines, lungs were fixed in $3.7 \%$ formaldehyde, in $0.1 \mathrm{M}$ PBS, $\mathrm{pH} 7.25 ; 7 \mu \mathrm{m}$ sections were cut in a cryostat. 


\section{Enzyme assay:}

a) Determination of exoglycosidase activity

For enzymatic activity, lung rudiments and fibroblasts were homogenized in $2 \mathrm{ml}$ (tissue) or in $1 \mathrm{ml}$ (fibroblasts) of $0.1 \mathrm{M}$ citrate/ $0.2 \mathrm{M}$ phosphate buffer, $\mathrm{pH} 4.5$, then centrifuged at 25,000 $\mathrm{g}$ for 10 min and the supernatants were used. In preliminary experiments, no enzymatic activity was detected in the sediments. $\mathrm{B}$-N-acetyl-glucosaminidase (EC 3.2.1.30), B-D-glucuronidase (EC 3.2.1.31), B-Nacetyl-galactosaminidase (EC 3.2.1.53) and Bgalactosidase (EC 3.2.1.23) were assayed as previously described by Orlacchio et al. (1984) using, respectively, as substrates 4-methyl-umbelliferyl- $\beta$ $\mathrm{N}$-acetyl glucosaminide, 4-methyl-umbelliferyl- $\beta$ D-glucuronide, 4-methyl-umbelliferil- $\beta$-N-acetylgalactosaminide 4-methyl-umbelliferyl-ß-galactoside in $0.1 \mathrm{M}$ citrate $/ 0.2 \mathrm{M}$ phosphate buffer, $\mathrm{pH}$ 4.5 , and $50 \mu 1$ of enzyme preparation.

One enzyme unit is defined as the amount of the enzyme that converts $1 \mathrm{nmol} / \mathrm{hr}$ of substrate into 4methyl-umbelliferone at $37^{\circ} \mathrm{C}$. Specific activity is expressed as units/mg protein. Proteins were determined according to the Bradford method (1976) using cristalline calf gamma globuline (Merck) as standard.

\section{b) Determination of HAase activity}

Whole lung rudiments, various lung regions and fibroblasts cultured as described above were sonicated on ice in formate extraction buffer $(0.1 \mathrm{M}$ sodium formate/ $0.15 \mathrm{M} \mathrm{NaCl} / 0.1 \%$ Triton X-100, $\mathrm{pH} 3.5$ ) containing $2.5 \mathrm{mM}$ saccharic acid 1,4-lactone, an inhibitor of exoglycosidase activity, according to the method of Kulyk and Kosher (1987). Aliquots of the sonicate were used for protein determination (Bradford 1976). The sonicate was centrifuged at $10,000 \mathrm{~g}$ for $5 \mathrm{~min}$ and aliquots of the supernatant were incubated at $37^{\circ} \mathrm{C}$ for $18 \mathrm{hr}$ with $200 \mu \mathrm{g}$ HA (Sigma -Aldrich, Milan, Italy) in $250 \mu 1$ reaction mixture. HAase activity was measured by assaying the formation of reducing terminal N-acetyl-glucosamine-containing oligosaccharides by a micromodification of the procedure of Reissig et al., (1955). Briefly, the $250 \mu$ l reaction mixtures were evaporated to dryness, and the residue was dissolved in $70 \mu \mathrm{l}$ of $0.125 \mathrm{M}$ potassium tetraborate and heated in a boiling water bath for exactly $3 \mathrm{~min}$. After cooling, the samples were added with $330 \mu \mathrm{l}$ of diluted dimethylaminobenzaldehyde reagent (Reissig et al., 1955) and incubated at $37^{\circ} \mathrm{C}$ for $20 \mathrm{~min}$. At the end of the incubation, samples were cooled in an ice bath and centrifuged at $10,000 \mathrm{~g}$ for $5 \mathrm{~min}$, and the adsorbance of supernatants was measured at $585 \mathrm{~nm}$. For controls, supernatants of tissue extracts were heat-inactivated by boiling before addition of HA substrate. Terminal $\mathrm{N}$-acetylglucosamine was not detectable in these control samples.

HAase activity was determined in medium and fibroblast culture with two assays, at $\mathrm{pH} 3.5$ and 6.5. HAase activity is expressed as units/mg protein. Units of HAase are defined as nmol of terminal Nacetylglucosamine released per hr at $37^{\circ} \mathrm{C}$.

\section{Histochemical localization}

We localised $\beta$-NAG and $\beta$-GLU enzymes in embryonic chick whole lungs at incubation days 7 , 9, 11, 13 and 18. The B-NAG staining was assayed according to Hayashi's method (1965). Briefly, sections were immersed in $0.1 \mathrm{M} \mathrm{Na}$-citrate buffer, $\mathrm{pH}$ 5.2, containing $0.25 \mathrm{mg} / \mathrm{ml}$ Fast-Garnet GBC (Sigma-Aldrich, F-8761) and $0.2 \mathrm{mg} / \mathrm{ml}$ naphthol ASBI N-acetyl-B-D-glucosaminide (Sigma, N-4006) for $1 \mathrm{~h}$ at room temperature. Some lungs were stained in toto for B-NAG. The B-GLU staining was assayed as described by Pipe and Moore (1985); the sections were immersed in $0.1 \mathrm{M} \mathrm{Na}$ acetate-acetic acid buffer $\mathrm{pH} 4.5$, containing $0.3 \mathrm{mg} / \mathrm{ml}$ Fast-Red GG (Sigma, F-7013) and $0.28 \mathrm{mg} / \mathrm{ml}$ naphthol ASBI N-acetyl- $\beta$-D-glucuronic acid (Sigma, N-1875) for $3 \mathrm{~h}$ at $37^{\circ} \mathrm{C}$. Controls of $\beta-\mathrm{NAG}$ reaction were determined in $0.1 \mathrm{M}$ Na-citrate buffer $\mathrm{pH}$ 5.2; controls of $\beta$-GLU were determined in $0.1 \mathrm{M} \mathrm{Na}$ acetate-acetic acid buffer, $\mathrm{pH} 4.5$.

\section{Immunohistochemistry}

For CS analysis, a monoclonal mouse antibody (Bio Makor, Kiryat Weizmann, 76326 Rehovot, Israel, cod. 6505) diluted 1:100 was utilized. The secondary antibody against mouse $\mathrm{IgG}$ was conjugated with alkaline phosphatase (Sigma, A-3688). For HA analysis, we used a probe constituted by hyaluronectin, a glycoprotein extracted from brain by Delpech et al. (1991) which is able to bind to HA and not to other GAG. The hyaluronectin conjugated with alkaline phosphatase was provided by Girard Nicole (Centre Henri Becquerel-Roven) and utilized as described by Marret et al. (1994). 
For HS analysis, a primary polyclonal rabbit anti HS antibody diluted 1:250 (kindly supplied by Patricia Simon-Assmann, Inserm, Strassburg) was utilized. For $\mathrm{TGF}_{2}$ localization, we used $\mathrm{Rb} 34$ antibody obtained from rabbits immunized against a synthetic peptide constituted by the first 29 aminoacids of $\mathrm{TGF}_{2}$, supplied by Celtrix Laboratories (Palo Alto, USA), diluted 1:40. IL-1 was localized by rabbit anti-IL-1 antibody (Genzyme, Milan, Italy, EP-200) diluted 1:40. The secondary antibody against the primary rabbit antibody was a biotinylated goat anti-rabbit IgG (Bio-Division, Milan, Italy, R001-63), diluted 1:250. The third molecule conjugated with peroxidase was streptavidin (Biodivision, G014-63). For peroxidase visualization, diaminobenzidine was used (Dako Corporation, CA 93013, USA, S 3000) (Orlacchio et al., 1984). For alkaline phosphatase, Fast-Red (Sigma, F-5146) and phosphate naphthol AS-TR (Sigma, N-8518) were used (Orlacchio et al., 1984).

\section{Differential reactions for glycosidase, GAG and HA or CS}

We also performed on 9-day-old lung sections a sequential reaction for B-NAG and for HA and CS respectively.

Control sections for each reaction were also performed, omitting primary antibody.

\section{Fibroblast cultures}

Lung rudiments were removed under sterile conditions from chick embryos at incubation days 7, 9, 11,13 and 18 . Tissues were pooled (70 embryos at day of incubation 7; 30 at days 9 and 11; 10 at days 14 and 18), cut in small pieces and dissociated in $0.25 \%$ trypsin (Difco Laboratories, Detroit, Mich., 1/250) in $\mathrm{Ca}^{++}$- and $\mathrm{Mg}^{++}$-free Hanks balanced salt solution for $20 \mathrm{~min}$ at room temperature. The dissociated cells were filtered through a nylon mesh, centrifuged ( $35 \mathrm{~g}, 10 \mathrm{~min}$ ), then washed with phosphate buffered saline (PBS), pH 7.4 and suspended in medium 199 (Gibco, Grand Island, NY) plus 10\% fetal calf serum (Gibco, Grand Island, NY). $15 \mathrm{ml}$ of the cell suspension $\left(1 \times 10^{6} \mathrm{cell} / \mathrm{ml}\right)$ was plated in $75 \mathrm{~cm}^{2}$ Falcon plastic flasks in humidity-saturated atmosphere $\left(5 \% \mathrm{CO}_{2}, 37^{\circ} \mathrm{C}\right)$ for $24 \mathrm{hrs}$, to enable confluent and stationary fibroblast cultures. Subsequently, nutrient was exchanged, the cells were washed with PBS, added with medium 199 alone and incubated for $24 \mathrm{hrs}$. At the end of the incubation period, the cells were washed with physiologi- cal saline, recovered by gently scraping the monolayer with $1 \mathrm{ml}$ of $10 \mathrm{mM}$ citrate buffer, $\mathrm{pH} \mathrm{6}$, and then used for enzymatic determination. Viability was measured by the cells' ability to exclude trypan blue (Patterson, 1980). The number of viable cells/culture was determined at the incubation time using a Bürker chamber.

\section{Statistical analysis}

Statistical analysis was performed using the student $t$ test for paired and unpaired data and the difference was considered significant at 0.05 level.

\section{RESULTS}

\section{Lung rudiments}

The main stages of temporally related embryonic lung development are shown in Fig. 1 (drawing modified from Romanoff, 1960). For further morphological specifications refer to Becchetti et al. (1988).

\section{Glycosidase biochemistry}

In Table I, the HAase, $\beta$-NAG, $\beta$-NAcGALA, $\beta$ GALA and B-GLU activities in embryonic chick whole lungs of the developmental days considered are listed. HAase, B-NAG and B-NAcGALA show constant increases in specific activity from developmental day 7 to $11(\mathrm{p}<0.01)$, then they decrease $(\mathrm{p}<0.01)$ till the $18^{\text {th }}$ development day. In contrast, B-GLU continues to increase until day 11 and maintains high values until day 18 , whereas $B$ GALA shows high activity at developmental days 7, 11 and 18, with decreases at days 9 and 13 .

\section{Spatial determinations}

\section{Apical lung region}

In the apical lung region (Table II), HAase, BNAG, B-NAcGALA and B-GLU produce the highest activity at day $11(\mathrm{p}<0.01)$ and subsequently decrease progressively until day 18 ; conversely, $B$ GALA shows high activity at developmental day 7 , decreases up to day 13 and subsequently increases again at developmental day 18.

\section{Medial lung region}

In the medial lung region (Table II), HAase shows the same trend as in the apical area, while B-NAG, $\beta$-NAcGALA and $\beta$-GLU increase signif- 


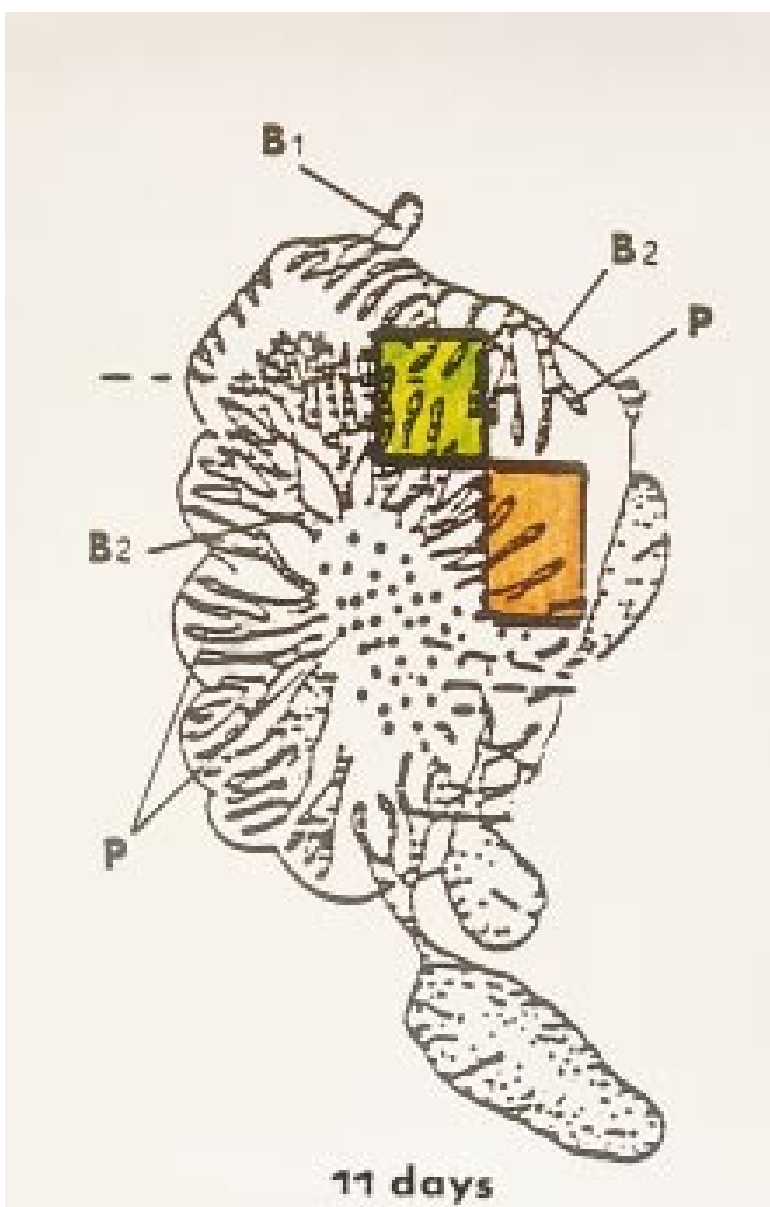

Fig. 1 - 11-day-old chick embryo lung is depicted. B1 represents the primary bronchus or mesobronchus; B2 the secondary bronchi; $\mathrm{P}$ the parabronchi (or tertiary bronchi). The area indicated by the green rectangle is explicative for figures 7 and 8 , the area indicated by the orange rectangle is explicative for figure 9 . icantly until day 11, then decrease at day 13 and increase again at developmental day 18. B-GALA, instead, increases its specific activity constantly from developmental day 7 to 18 .

\section{Caudal lung region}

In the caudal lung region (Table II), HAase, $\beta$ NAG, B-NAcGALA and B-GLU enzymes show the same trend as the previously examined region, while B-GALA shows a constant increase until day 11 , then decreases at day 13 and increases again at developmental day 18 .

\section{Glycosidase histochemistry}

We examined the localisation of $\beta-N A G$ and $\beta-$ GLU in lungs on developmental days 7, 9, 11, 13 and 18 as well as the spatial distribution of glycosidases and their colocalisation with GAG and growth factor or cytokine in a section of embryonic chick lung on 9 day.

With regard to B-NAG (Figs. 2-4-7-8 and 10) and B-GLU (Figs. 3-5-9 and 11), we observed that the two enzymes are more active in the cell compartment than in the extracellular compartment. B-NAG shows greater staining than B-GLU. In epithelial cells, the two enzymes accumulate mainly in Golgi zone (Figs. 4 and 5) and in the laterobasal regions of the cell (Fig. 7). The highest degree of reactivity was observed in the more recent areas, epithelium of $2^{\text {nd }}$ order bronchi $\left(\mathrm{B}_{2}\right)$ (at day 7, Figs. 2 and 3), then in $3^{\text {th }}$ order bronchi or parabronchi (P) (at day 9, Figs. 4 and 5) and at day 11 (Figs. 6, 7 and 8), and finally at the level of aeriphery capillaries (a.c.) while they are developing at day 13 (Figs. 10 and 11). The

Table I

Specific activity of HAase, $\beta-N A G, \beta-G L U, \beta-N-A c G A L A$, GALA, at lung development days 7, 9, 11, 13 and 18 . The values are the means \pm SD of five independent experiments

\begin{tabular}{lccccc}
\hline $\begin{array}{l}\text { Lung } \\
\text { development } \\
\text { (day) }\end{array}$ & \multicolumn{5}{c}{ Specific activity (units/mg protein) } \\
\cline { 2 - 6 } & HAase & B-NAG & B-NacGALA & B-GALA & B-GLU \\
\hline 7 & $1.28 \pm 0.41$ & $368.4 \pm 43.5$ & $55.7 \pm 6.5$ & $224.2 \pm 33.6$ & $102.1 \pm 22.2$ \\
9 & $1.75 \pm 0.36$ & $490.3 \pm 58.6$ & $75.9 \pm 9.7^{*}$ & $142.3 \pm 26.6^{*}$ & $108.3 \pm 15.7$ \\
11 & $1.99 \pm 0.50^{*}$ & $1028.0 \pm 123.4^{*}$ & $181.4 \pm 20.1^{*}$ & $184.5 \pm 35.1$ & $172.7 \pm 28.6^{*}$ \\
13 & $1.11 \pm 0.32$ & $621.3 \pm 74.5^{*}$ & $101.5 \pm 11.8^{*}$ & $125.0 \pm 20.7^{*}$ & $131.7 \pm 24.5$ \\
18 & $0.51 \pm 0.04^{*}$ & $582.7 \pm 65.5^{*}$ & $104.9 \pm 12.8^{*}$ & $227.6 \pm 30.3$ & $163.0 \pm 30.0^{*}$ \\
& & & & &
\end{tabular}

$* \mathrm{P}<0.01$ vs development day 7 . 

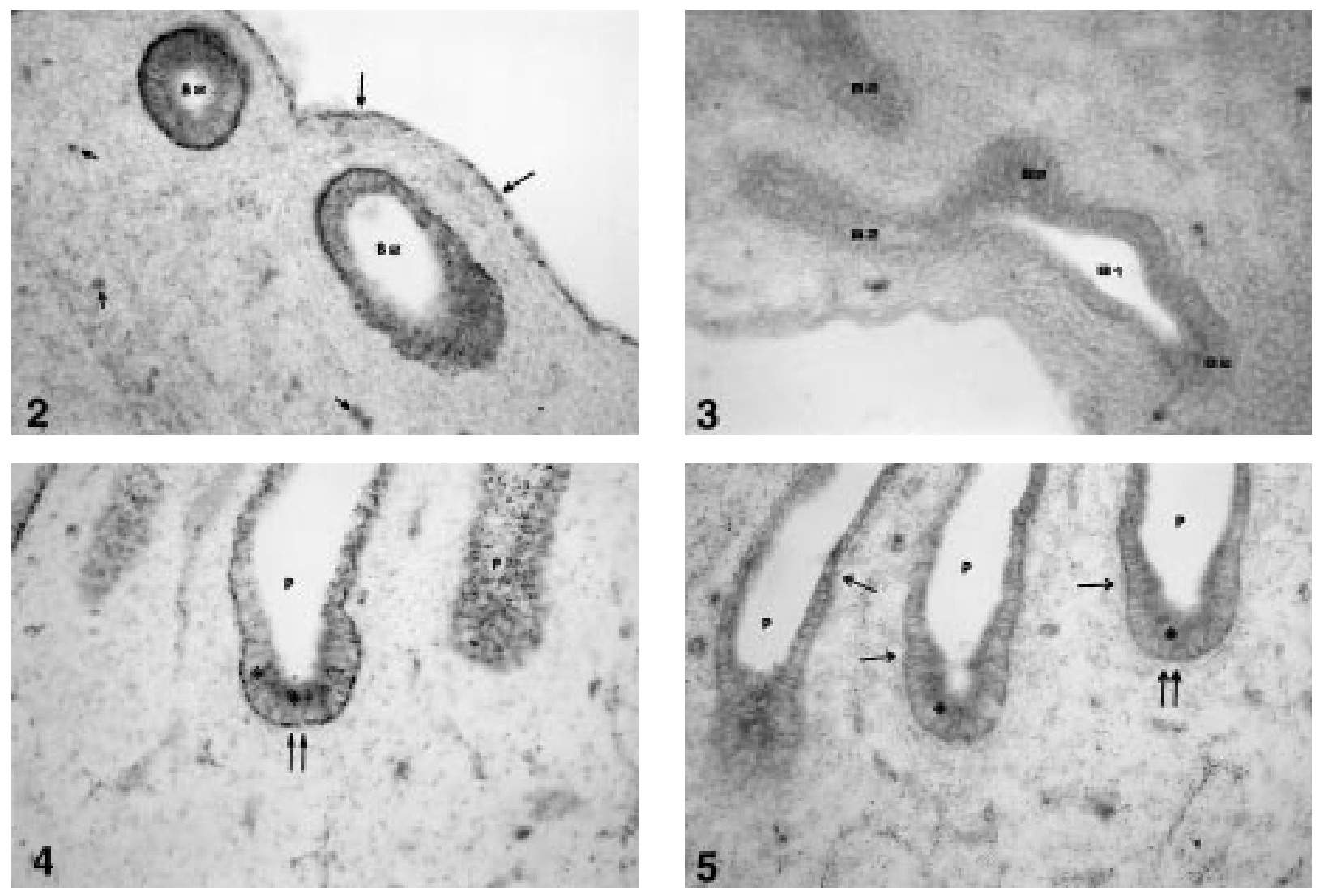

Figs. 2/5 - (2)7-day-old chick embryo lung. B-NAG reaction. Strong reactivity is shown in the epithelial basal side of B2. Long arrows indicate positivity in the pleuric layer; short arrows indicate positivity in some mesenchymal cells (likely macrophages or mast cells). Magnification, 450x. (3)7-day-old chick embryo lung. B-GLU reaction. Evident reactivity (less than that for B-NAG) is shown in B2 and B1. Magnification, 450x. (4)9-day-old chick embryo lung. B-NAG reaction. Two P are shown. Double arrows indicate prevalent positivity in the epithelial basal side; asterisks in the Golgi areas. Magnification, 450x. (5)9-day-old chick embryo lung. B-GLU reaction. As for B-NAG, B-GLU positivity is localized in the Golgi areas of parabronchial epithelium (double arrows). Positivity is evident also in basal membranes (single arrow), mainly located at the growth fronts (asterisks). Magnification, 450x.

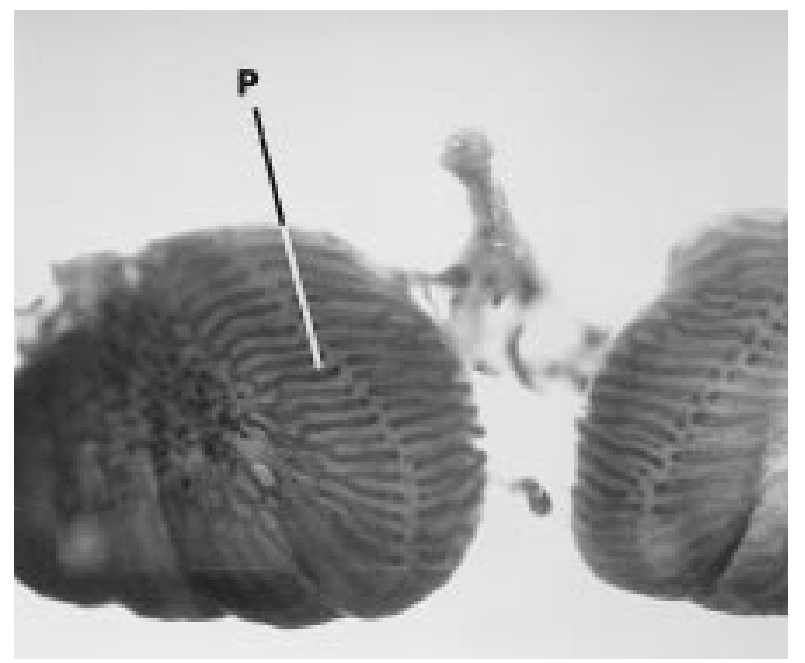

positivity is shown especially on the growth front and in the surrounding mesenchymal area (Figs. 4, 5,7 and 8). Strong intensity was also found in some cells of surrounding mesenchyme (probably macrophages and mast-cells) (Figs. 2, 7 and 9) and in the mesothelial cells of external side (Fig. 2), while less intensity was observed in the vessel endothelium (Fig. 10). Histochemical controls of $\beta$ NAG and $\beta$-GLU reactions were negative.

\section{GAG immunohistochemistry}

CS proteoglycans are evident in the basal membranes and in the intermediate mesenchyme

Fig. 6 - 11-day-old chick embryo lung. B-NAG reaction in organs stained in toto. Clear positivity in $\mathrm{P}$, prominently in growth fronts. Magnification, $17 x$. 
Table II

Glycosidase specific activity in apical, medial, and caudal region of chick embryo lung at different development stages (days 7, 9, 11, 13, 18)

\begin{tabular}{|c|c|c|c|c|c|}
\hline \multicolumn{6}{|c|}{ Specific activity (units/mg protein) } \\
\hline \multirow[t]{2}{*}{ Enzyme } & \multicolumn{5}{|c|}{ Days } \\
\hline & 7 & 9 & 11 & 13 & 18 \\
\hline \multicolumn{6}{|c|}{ Apical region } \\
\hline HAase & $1.14 \pm 0.04$ & $1.90 \pm 0.08 *$ & $2.21 \pm 0.06^{*}$ & $1.11 \pm 0.07$ & $0.51 \pm 0.05^{*}$ \\
\hline ß-NAG & $368.4 \pm 43.5$ & $525.7 \pm 68.5^{*}$ & $857.0 \pm 97.7 *$ & $690.2 \pm 98.6^{*}$ & $712.5 \pm 88.9 *$ \\
\hline ß-NAcGALA & $55.7 \pm 6.5$ & $74.3 \pm 10.6^{*}$ & $138.5 \pm 20.9 *$ & $121.0 \pm 15.3 *$ & $125.3 \pm 17.5^{*}$ \\
\hline ß-GALA & $224.3 \pm 27.0$ & $172.3 \pm 20.3$ & $195.5 \pm 25.5$ & $128.0 \pm 14.8 *$ & $246.5 \pm 26.7$ \\
\hline ß-GLU & $102.4 \pm 12.3$ & $112.4 \pm 15.4$ & $205 \pm 20.6^{*}$ & $167.0 \pm 21.7 *$ & $162.6 \pm 20.4 *$ \\
\hline \multicolumn{6}{|c|}{ Medial region } \\
\hline HAase & - & $1.68 \pm 0.05^{\circ}$ & $1.98 \pm 0.05 * \circ$ & $1.20 \pm 0.04 * \circ$ & $0.48 \pm 0.02 *$ \\
\hline ß-NAG & - & $677.6 \pm 88.7^{\circ}$ & $985.0 \pm 96.9 * \circ$ & $876.5 \pm 96.6^{*}$ & $929.2 \pm 99.2 * \circ$ \\
\hline ß-NAcGALA & - & $115.7 \pm 17.7$ & $155.1 \pm 20.1 * \circ$ & $149.7 \pm 18.0^{\circ}$ & $162.2 \pm 19.4^{*}$ \\
\hline ß-GALA & - & $127.9 \pm 14.4^{\circ}$ & $137.5 \pm 6.6^{\circ}$ & $158.7 \pm 12.1 * \circ$ & $229.3 \pm 18.7 * \circ$ \\
\hline ß-GLU & - & $103.7 \pm 12.3$ & $209.0 \pm 30.1 *$ & $175.2 \pm 22.8^{*}$ & $210.2 \pm 23.1 * \circ$ \\
\hline \multicolumn{6}{|c|}{ Caudal region } \\
\hline HAase & $0.93 \pm 0.05^{\circ}$ & $1.59 \pm 0.07 * \circ$ & $1.76 \pm 0.02 * \circ$ & $0.92 \pm 0.05^{\circ}$ & $0.52 \pm 0.02 *$ \\
\hline ß-NAG & $368.4 \pm 43.5$ & $441.3 \pm 58.7$ & $981.5 \pm 99.4 *$ & $735.6 \pm 93.2 *$ & $852.0 \pm 96.7 *$ \\
\hline ß-NAcGALA & $55.7 \pm 6.5$ & $86.0 \pm 13.3^{*}$ & $186.1 \pm 23.2 * \circ$ & $128.7 \pm 21.0 *$ & $144.2 \pm 29.4^{*}$ \\
\hline ß-GALA & - & $120.0 \pm 16.9^{\circ}$ & $189.0 \pm 14.8^{*}$ & $166.8 \pm 15.7 * \circ$ & $224.3 \pm 20.2 *$ \\
\hline ß-GLU & - & $104.7 \pm 13.5$ & $172.6 \pm 22.4^{*}$ & $168.0 \pm 22.4^{*}$ & $204.4 \pm 23.5^{*}$ \\
\hline
\end{tabular}

$* \mathrm{P}<0.01$ vs day $7 ;{ }^{\circ} \mathrm{P}<0.01$ vs apical region

between tubuli (where the interlobular septa and vascular network envelope) or in the area near the basal membrane along the lateral sides of the bronchus (Fig. 12). HA accumulates in the areas near the basal membranes where CS are present either in the mesenchyme situated on the growth front of the bronchus, or in the most external areas of the lung bud in expansion (Fig. 13). HS proteoglycan appears constantly at the level of the basal membrane of each bronchus, except for that of the epithelial growth front.

In the experiments with $\mathrm{B}-\mathrm{NAG} / \mathrm{CS}$ or $\beta$ NAG/HA sequential reaction, HA and CS are less reactive in the area near the one where the enzyme is more expressed (Figs. 15 and 16). Immunohistochemical controls were negative.
Growth factor and cytokine immunolocalisation As concerns growth factor localisation, $\mathrm{TGF}_{2}$ is at the front where the sub-epithelial area changes continuously (Fig. 14), as observed along the basal membrane and in the sub-epithelial region of $2^{\text {nd }}$ and $3^{\text {th }}$ order bronchi, with greater intensity in lateral growing regions than in apical one (Fig 17). Also cytokine IL-1 immunostaining appears along the basal membrane, but shows higher intensity at the growth front of the bronchial epithelium, and less intensity along the lateral sides of the epithelial tubulus (Fig. 18).

On the whole $\mathrm{TGF}_{2}$ and $\mathrm{CS}$ are expressed in the same regions, while IL-1 is essentially correlated to HA. 

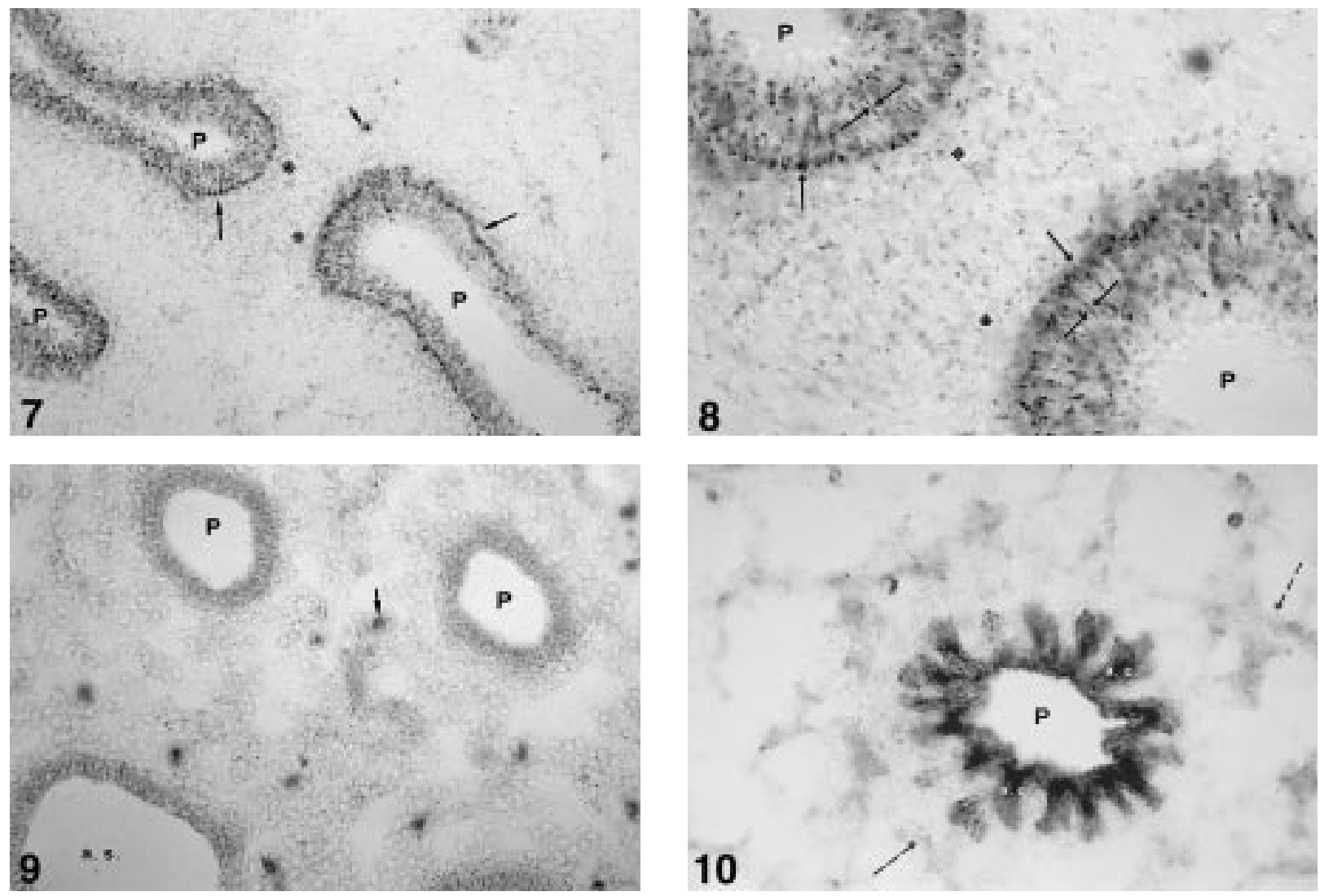

Figs. 7/10 - (7)11-day-old chick embryo lung. B-NAG reaction. Two parabronchi converge, indicating strong positivity in the epithelial growth fronts (long arrows). The asterisks correspond to interposed mesenchyme. Magnification, 450x. This figure corresponds to the area included within the green rectangle in Fig. 1. (8)11-day-old chick embryo lung B-NAG reaction. The greater magnification evidences strong positivity in both basal (single arrows) and lateral (opposite arrows) regions of P. The asterisks show the positivity also in the underlying mesenchymal ECM. Magnification, 1000x. This figure corresponds to the area included within the green rectangle in Fig. 1. (9)11-day-old chick embryo lung. B-GLU reaction. Positivity in the epithelial basal side of $\mathrm{P}$, air sacs (a. s.) and scattered mesenchymal cells (short arrow). Magnification, 450x. This figure corresponds to the area included within the orange rectangle in Fig. 1. (10)13-day-old chick embryo lung. B-NAG reaction. Very strong positivity of air capillaries (a. c.) emerging from the P. Dotted arrows indicate a weak intensity in vessel walls. Magnification, 450x.

\section{Fibroblast cultures}

Table III summarises HAase values in cultured fibroblasts of chick embryos on day 12 . Specific activity is evident both in cells and in culture media. Activity increases significantly in the extracellular region at $\mathrm{pH} 6.5$ (more than at $\mathrm{pH} 3.5$ ), while it decreases in the intracellular compartment $(\mathrm{p}<0.01)$, demonstrating that, at neutral $\mathrm{pH}$, a prevalent degradative action exists in the ECM close to the cell.

Table IV shows B-NAG and B-GLU activities in fibroblast cultures derived from lungs of developmental days $7,11,13,18$. B-NAG shows higher activity than $\beta-G L U$, while $\beta-N A G$ and $\beta-G L U$ display a similar trend. Glycosidase activity increases $(\mathrm{p}<0.01)$ in fibroblasts until incubation day 13 and then decreases, which is similar to the biochemical data for the whole lung.

\section{DISCUSSION}

In previous studies, we have demonstrated that a specific composition of ECM fulfils a regulatory role on the bronchial branching in chick embryos. This ECM composition shows a peculiar spatialtemporal distribution at the bronchial level during development (Becchetti et al., 1988). Therefore, it is clear that ECM remodelling processes play an important morphogenetic role. In this respect, it 
Table III

HAase activity in cultured fibroblasts from 12-day-old chick embryo lung maintained for 24 hrs without serum.

The values are the mean \pm SD of five independent experiments

HAase activity (units/mg protein)

\begin{tabular}{ccccc}
\hline Time & \multicolumn{2}{c}{ Fibroblast } & \multicolumn{2}{c}{ Culture medium } \\
\hline Hours & $\mathrm{pH} 3.5$ & $\mathrm{pH} 6.5$ & $\mathrm{pH} 3.5$ & $\mathrm{PH} 6.5$ \\
3 & $1.49 \pm 0.16$ & - & $0.02 \pm 0.003$ & $0.07 \pm 0.008^{*}$ \\
24 & $1.40 \pm 0.17$ & $0.26 \pm 0.03^{*}$ & $0.06 \pm 0.007^{\circ}$ & $0.14 \pm 0.002^{*}$ \\
\hline
\end{tabular}

$* \mathrm{P}<0.01$ vs $\mathrm{pH} 3.5 ;{ }^{\circ} \mathrm{P}<0.01$ vs $\mathrm{pH} 3.5$ at 3 hrs.
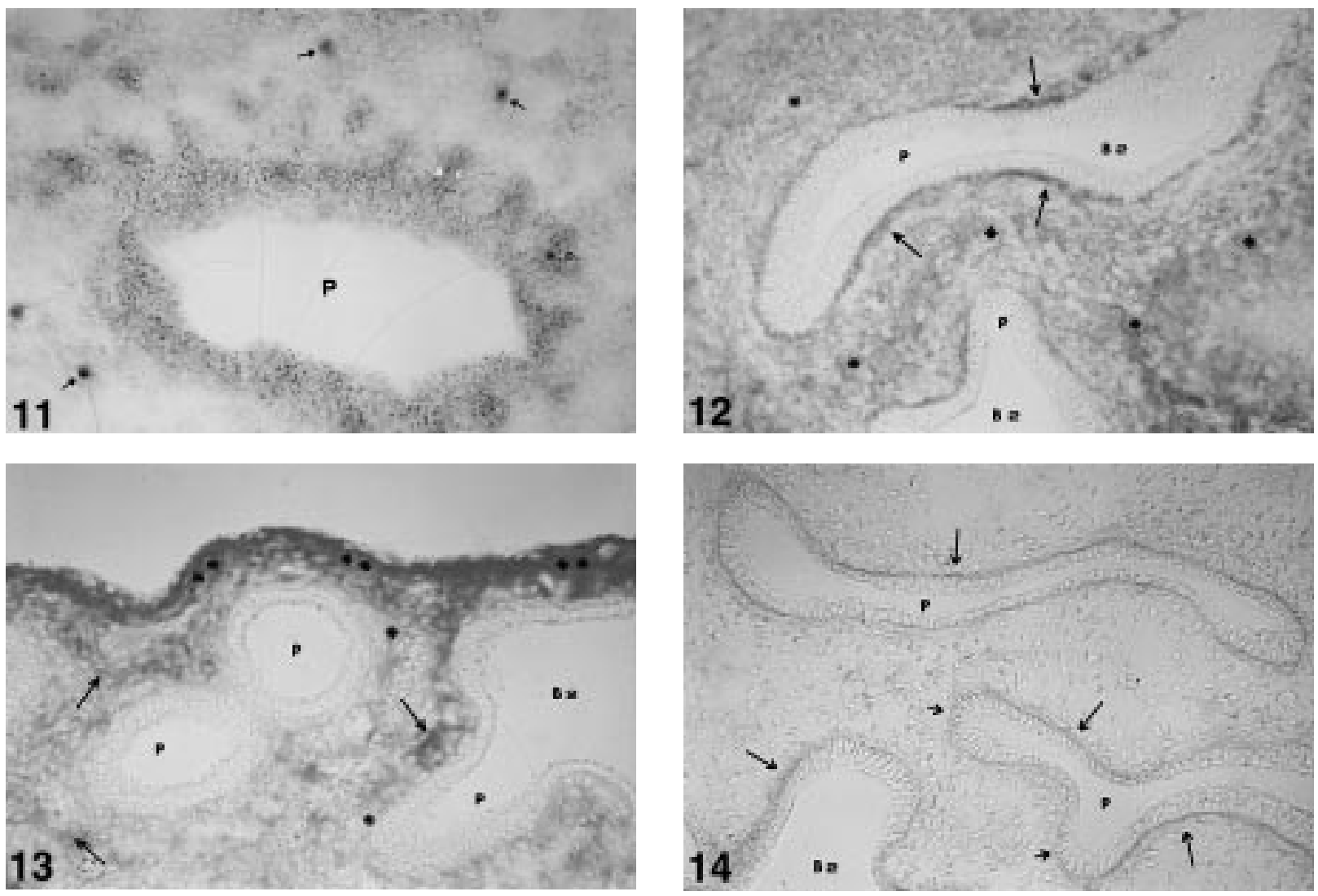

Figs. 11/14 - (11)13-day-old chick embryo lung. B-GLU reaction. Clear positivity of air capillaries (a. c.) and mesenchymal cells (arrows). Magnification, 450x. (12)9-day-old chick embryo lung. CS immunohistochemistry. Strong positivity for CS in the mesenchymal ECM underlying the epithelial wall where P emerge from B2 (long arrows), and in the intermediate mesenchymal areas (asterisks). Magnification, 450x. (13)9-day-old chick embryo lung. Reaction for HA. HA positivity predominates around the emergence of $\mathrm{P}$ (long arrows); a very intense reactivity in newly-forming mesenchymal areas underlying the pleura can been seen at the double asterisks. Single asterisk indicates mesenchymal areas, which are weakly marked (due probably to the degradative action of glycosidases). Magnification, 450x. (14)9-day-old chick embryo lung. HS immunohistochemistry. HS positivity is localized in the ECM underlying the bronchial epithelial layer (long arrows). Lesser reactivity is seem in growth fronts (short arrows). Magnification, 450x. 

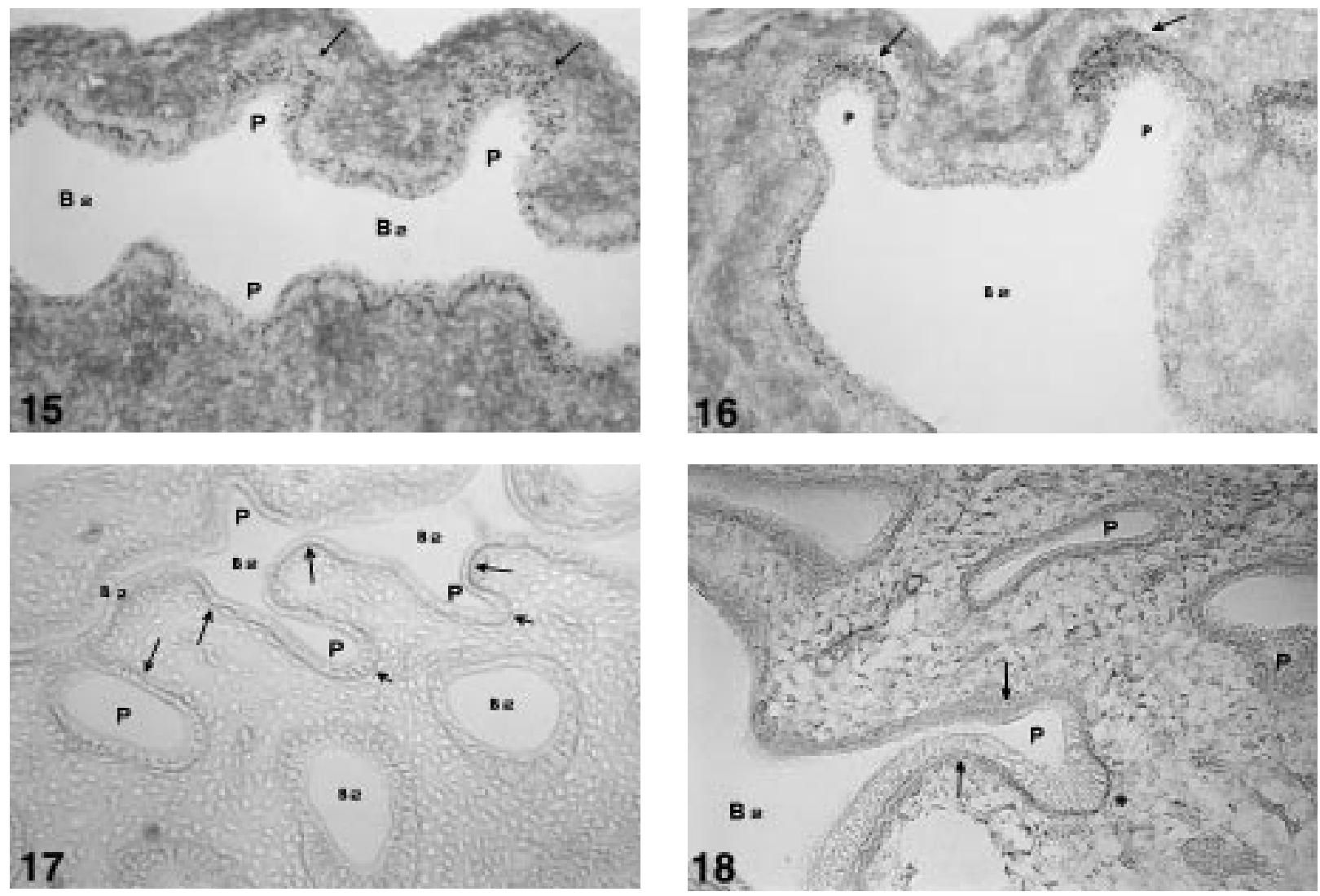

Figs. 15/18 - (15)9-day-old chick embryo lung. B-NAG + CS double reaction. B-NAG reactivity is prevalently located in epithelium of parabronchial growth fronts. Underlying mesenchymal ECM evidences less positivity for CS (arrows). Magnification, 450x. (16)9-day-old chick embryo lung. B-NAG + HA double reaction. Similar to Fig. 15, the greater ß-NAG reactivity correlates to decreased HA staining around parabronchial growth fronts (arrows). Magnification, 450x. (17)9-day-old chick embryo lung. $\mathrm{TGF} \beta_{2}$ immunostaining. Prevalent $\mathrm{TGF}_{2}$ staining is located in $\mathrm{ECM}$ underlying the bronchial epithelium, mainly in the areas interposed between adjacent $\mathrm{P}$ and along their lateral walls (long arrows). Note a lesser staining in correspondence of the growth front of $\mathrm{P}$ (short arrows). Magnification, 450x. (18)9-day-old chick embryo lung. IL-1 immunostaining. IL-1 staining is located in ECM (inclusive of the basement membrane) underlying the bronchial epithelium (long arrows) but, unlike $\mathrm{TGF}_{2}$, strong IL-1 staining is evident around the epithelial growth front of P (asterisk). Magnification, 450x.

has been demonstrated that exogenous GAG are able to modify glycosidase activity (Hoodless and Wrana, 1998; Bodo et al., 1991).

In the present studies, we investigated the spatialtemporal distribution of certain glycosidases in relation to the spatial distribution of GAG, a PG, a growth factor and a cytokine, in order to outline the changes on ECM composition, the involved processes and any other correlation with in vitro lung development.

Our data show an increase of HA in the epithelial branching growth front, while CS increases in the lateral side of epithelial branching. At the same time, the concentration of degradative enzymes shows different concentrations of $B-N A G$ and $B-$
GLU in different lung regions; their significant increase during lung development demonstrates that fibroblasts can be differently activated according to spatial and temporal induction. This significant increase in glycosidase activity is also confirmed in vitro in cultured fibroblasts derived from 11-day-old developmental lungs. In particular, the increase in $\beta-N A G$ and $B-G L U$ activities in the growth front of the bronchial tubule would cause a CS decrease and an HA increase, while in the lateral regions B-NAG activity induces an HA decrease and a CS increase.

ECM degradation depends on the release of growth factors. Substances such as $\mathrm{TGF}_{2}$ and IL1 play a key role in controlling the processes of 
Table IV

Specific activity of $\beta-N A G$ and $\beta-G L U$ (units/mg protein) in cultured fibroblasts from lung of development days 7 , 11, 13 and 18 maintained for 24 hrs without serum. The values are the mean \pm SD of five independent experiments

\begin{tabular}{lcccc}
\hline \multirow{4}{*}{ Enzyme } & \multicolumn{4}{c}{ B-NAG and $\boldsymbol{\beta}-\mathbf{G L U}$ specific activity $($ units/mg protein) } \\
\cline { 2 - 5 } & day $\mathbf{7}$ & day $\mathbf{1 1}$ & day $\mathbf{1 3}$ & day $\mathbf{1 8}$ \\
\hline B-NAG & $168.7 \pm 13.5$ & $230.6 \pm 16.3^{*}$ & $330.2 \pm 19.8^{*}$ & $110.2 \pm 14.7^{*}$ \\
B-GLU & $7.1 \pm 0.9$ & $27.7 \pm 1.8^{*}$ & $48.5 \pm 7.1^{*}$ & $28.7 \pm 6.5^{*}$ \\
\hline
\end{tabular}

$* \mathrm{p}<0.01$ vs development day 7.

ECM proliferation, migration, differentiation, storage and organization. In fact $\mathrm{TGF}_{2}$ induces $\mathrm{ECM}$ synthesis (Locci et al., 1993), thus inhibiting its degradation (Edwards et al., 1987); conversely IL1 induces the production of ECM-degrading enzymes (Mauviel, 1993), inhibiting its synthesis (Bodo et al., 1994).

IL-1 is preferentially located in the epithelial growth front where HA, which favours cell migration, accumulates; while $\mathrm{TGF}_{2}$ is predominantly found along the lateral sides where CS and HS, respectively related to tissue stabilization and epithelial-mesenchymal interactions, are localized. Therefore, it is evident that HA accumulation occurs especially during proliferation and growth phases, and an increase in sulphated GAG is concomitant with processes of bronchial branching and organization of the interlobular septa. This is in agreement with findings by Caniggia and Post (1992), who demonstrated that rat lung fibroblasts produce HA in proximity to the epithelium, and that increases in HS or CS coincide with a thinning of the alveolar septal wall.

In conclusion, our experimental data provide specific information on ECM remodelling processes and on some aspects of control mechanisms.

The more dynamic regions are found to be located in the epithelial growth front, while the lateral regions are confirmed as stabilization sites; in fact, glycosidases increase on the growth front of bronchial buds. At the level of this front HA prevails, while CS are more concentrated in the lateral regions. Likewise, $\mathrm{IL}-1$ is more expressed in the growth front, as opposed to $\mathrm{TGF}_{2}$ in the lateral regions. We have shown that IL-1 increases HA (Bodo et al., 1994) and TGFß CS synthesis (Locci

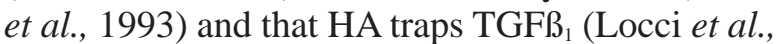

1995). This linkage could prevent the inhibiting action of growth factor on epithelial cell proliferation, so that the ECM proliferative stimulus could be accepted by epithelial branching cells. In addition, the HA decrease and consequent $\mathrm{TGF}_{2}$ release, and the CS increase in the lateral sides of epithelial branching could cause a blocking of proliferation and an epithelial stabilization. The action seems to be mediated by HS proteoglycan, because it plays an important biological role in cell-matrix adhesion processes and is a regulator of growth factor actions (Miao et al., 1997).

Our results enable us to hypothesize a probable sequence of events : degradative activity of glycosidases, changes in ECM composition, altered activity of growth factors and cytokines, and stimulation of cell proliferative, migratory, and differentiative processes.

\section{ACKNOWLEDGMENTS}

These studies were supported by grants from the Ministero dell'Università e della Ricerca Scientifica e Tecnologica (MURST).

\section{REFERENCES}

Becchetti E., Evangelisti R., Stabellini G., Pagliarini A., Del Borrello E., Calastrini C., and Carinci P.: Developmental heterogeneity of mesenchymal glycosaminoglycans (GAG) distribution in chick embryo lung anlagen. Am. J. Anat. 181, 3342, 1988

Bodo M., Becchetti E., Pezzetti F., Marinucci L., Evangelisti R., and Carinci P.: Exogenous glycosaminoglycans modulate glycosidase activity in cultured embryonic fibroblasts. Med. Sci. Res. 19, 803-805, 1991. 
Bodo M., Becchetti E., Giammarioli M., Baroni T., Bellucci C., Pezzetti F., Calvitti M., and Carinci P. Interleukin-1 and interleukin-6 differentially regulate the accumulation of newly synthesised extracellular matrix components and the cytokine release by developing chick embryo skin fibroblasts. Int. J. Dev. Biol. 38, 535-542, 1994.

Bradford M.M.: A rapid and sensitive method for the quantitation of microgram quantities of protein utilizing the principle of protein-dye binding. Anal. Biochem. 72, 248-254, 1976.

Caniggia I., Tanswell K, and Post M.: Temporal and spatial differences in glycosaminoglycan synthesis by fetal lung fibroblasts. Exp. Cell Res. 202, 252-258, 1992.

Carinci P.: The mesenchyme during development: composition of the ground substance, regulatory factors, embryological significance. Bas. Appl. Histochem. 25, 267-277, 1981.

Carinci P., Becchetti E., Stabellini G., Evangelisti R., and Pagliarini A.: Evidence for glycosaminoglycans role in avian lung morphogenesis. In Progress in Development Biology, Part B. Alan R. Liss, Inc. pp. 385-395, 1986.

Davies J., Lyon M., Gallagher J., and Garrod D.: Sulphated proteoglycan is required for collecting duct growth and branching but not nephron formation during kidney development. Development 121, 1507-1517, 1995.

Delpech B., Maingonnat C., Delpech A., Maes P., Girard N., and Bertrand P.: Characterization of a hyaluronic acid-binding protein from sheep brain: comparison with human brain hyaluronectin. Int. J. Biochem. 23, 329-337, 1991.

Edwards D.R., Murphy G., Reynolds J.J., Whitham S.E., Docherty A.J.P., Angel P., and Heath J.K.: Transforming growth factor beta modulates the expression of collagenase and metalloproteinase inhibitor. EMBO J. 6, 1899-1904, 1987.

Evangelisti R., Valeno V., Bosi G., Bodo M., Scalabrini P., Stabellini G., Pezzetti F., and Carinci P.: A contribution to the regulation of proteoglycan production: modulation by TGF $\alpha$, TGFß and IL-1 of glycosaminoglycan biosynthesis on B-Dxyloside in chick embryo fibroblasts. Conn. Tissue Res. 37, 77-85, 1998.

Hamilton H.L.: Lillie's development of the chick. Holt, Rinehart and Winston, New York, 1965.

Hayashi M.: Histochemical demonstration of N-acetyl-ß-glucosaminidase employing naphthol AS-BI N-acetyl-B-glucosaminide as substrate. J. Histochem. Cytochem. 13, 355$360,1965$.

Hoodless P.A., and Wrana J.L.: Mechanism and function of signaling by the TGF $\beta$ superfamily. Curr. Topics Microbiol. Immunol. 228, 235-272, 1998.

Kulyk W.M. and Kosher R.A.: Temporal and spatial analysis of hyaluronidase activity during development of the embryonic chick limb bud. Dev. Biol. 120, 535-541, 1987.

Kresse H. and Glössl J.: Glycosaminoglycan degradation. In Advances in Enzymology. Meister A., (Ed.). John Wiley \& Sons, New York, 60, 217-311, 1987.
Locci P., Lilli C., Marinucci L., Baroni T., Pezzetti F., and Becchetti E.: Embryonic skin fibroblasts release TGF alpha and TGF beta able to influence synthesis and secretion of GAG. Cell Mol. Biol. (Noisy-le-grand) 39, 415-426, 1993.

Locci P., Marinucci L., Lilli C., Martinise D., Becchetti E.: Transforming growth factor $\beta$ 1-hyaluronic acid interaction. Cell Tissue Res. 281, 317-324, 1995.

Marret S., Delpech B., Delpech A., Asou H., Girard N., Courel M-N., Chauzy C., Maingonnat C., and Fessard C.: Expression and effects of hyaluronan and of the hyaluronanbinding protein hyaluronectin in newborn rat brain glial cell cultures. J. Neurochem. 62, 1285-1295, 1994.

Mauviel A.: Cytokine regulation of metalloproteinase gene expression. J. Cell Biochem. 53, 288-295, 1993.

Miao H-Q., Ornitz D.M., Aingorn E., Ben-Sasson S.A., and Vlodavsky I.: Modulation of fibroblast growth factor-2 receptor binding, dimerization, signaling, and angiogenic activity by a synthetic heparin-mimicking polyanionic compound. J. Clin. Invest. 99, 1565-1575, 1997.

Orlacchio A., Maffei C., Emiliani C., Rambotti P., and Davis S.: A distinct $B$-hexosaminidase isoenzyme separated from human leukemic lymphocytes and myelocytes. Biochem. Biophys. Res. Comm. 122, 966-973, 1984.

Patterson Jr. M.K.: Measurement of growth and viability of cells in culture. In: Jacoby W.B., Pastan I.H. (eds). Methods in Enzymology. Academic Press, New York, pp. 267-371, 1980.

Pipe R.K., and Moore M, N.: The ultrastructural localization of lysosomal acid hydrolases in developing oocytes of the common marine mussel Mytilus edulis. Histochem. J. 17, 939949, 1985

Reissig J.L., Strominger J.L., and Leloir L.F.: A modified colorimetric method for histological estimation of $\mathrm{N}$-acetylamino sugars. J. Biol. Chem. 217, 959-966, 1955.

Romanoff A.L.: The avian embryo. Structural and functional development. The Macmillan Company, New York, pp. 535567,1960

Stabellini G., Pellati A., Tosi L., Caruso A., and Carinci P.: Exogenous spermidine modulates glycosaminoglycan accumulation and epithelial differentiation in chick embryonic skin. J. Exp. Zool. 281, 594-601, 1998. 